\title{
Aspects of boundary conditions for non-Abelian gauge theories
}

\author{
A. P. Balachandran, ${ }^{1, *}$ V. P. Nair, ${ }^{2, \dagger}$ and Sachindeo Vaidya ${ }^{3, *}$ \\ ${ }^{1}$ Physics Department, Syracuse University, Syracuse, New York 13244-1130, USA \\ ${ }^{2}$ Physics Department, City College of the CUNY, New York, New York 10031, USA \\ ${ }^{3}$ Centre for High Energy Physics, Indian Institute of Science, Bangalore 560012, India
}

(Received 14 May 2019; published 6 August 2019)

\begin{abstract}
The boundary values of the time component of the gauge potential form externally specifiable data characterizing a gauge theory. We point out some of their consequences such as reduced symmetries, bulk currents for manifolds with disjoint boundaries and some nuances of how the charge algebra is realized.
\end{abstract}

DOI: 10.1103/PhysRevD.100.045001

\section{INTRODUCTION}

Boundary conditions on fields may be viewed as the idealization and simplification of the dynamics of these fields on the boundary of the spacetime region under consideration. They are also necessary, from a mathematical point of view, to make the problem well defined with the required self-adjointness properties for observables. Therefore, not surprisingly, the impact of boundary conditions on physical phenomena has been the subject of many investigations. In quantum field theory, the most extreme example of the importance of boundary conditions might be topological field theories where the entire dynamics is played out on the boundary [1]. Droplets of fermions in the quantum Hall effect, for which the effective theory is topological and the dynamics is reduced to that of the edge currents, is a physical realization of this [2]. A more standard, but still vivid, example of boundaries and their impact on bulk phenomena is the Casimir effect, which has been calculated for many different geometries and experimentally verified for many instances as well [3]. Among other examples of recent research interest we mention the possibility of edge states in gauge theories [4], bound states allowed by the general von Neumann theory of self-adjoint extensions [5,6], the role of boundary conditions and edge states for questions of entanglement in gauge theories [7], etc.

Closely related to the issue of boundary conditions is the asymptotic behavior of fields and its impact on realizations

\footnotetext{
*balachandran38@gmail.com vpnair@ccny.cuny.edu

¥vaidya@iisc.ac.in
}

Published by the American Physical Society under the terms of the Creative Commons Attribution 4.0 International license. Further distribution of this work must maintain attribution to the author(s) and the published article's title, journal citation, and DOI. Funded by SCOAP. of symmetry. It was recognized long ago that Lorentz transformations cannot be unitarily implemented on charged sectors of quantum electrodynamics due to the infrared behavior of the massless photon fields [8]. While this does not have any immediate impact on everyday laboratory applications of electrodynamics, since one can use the charge zero sector for such considerations, this does highlight nuances of how symmetry is realized in the theory. More recently similar effects have been analyzed in the context of gravitational fields. Infrared effects can also have an impact on the realization of symmetries other than Lorentz transformations as well. Obstructions to the unitary realization of color transformations or charge rotations in a non-Abelian gauge theory have been pointed out in [9]. Again, this may not have any immediate impact on calculations in the realistic case of QCD, for which the asymptotic states are expected to have zero color charge, but this does show the subtleties of symmetries in a gauge theory.

Another special feature is that, unlike theories of scalar or spinor fields, in a gauge theory, there is a field, namely the time component of the gauge potential $A_{0}$, whose sole effect is via its boundary values, the physical consequences of the bulk values of the field being wiped out by gauge invariance, i.e., by the Gauss law imposed on physical states. The complete elimination of $A_{0}$, as done, for example, in the so-called $A_{0}=0$ gauge, is too restrictive since the boundary value of this field is actually gauge invariant, in the sense of commuting with the constraints due to gauge symmetry. In this paper, we analyze this situation for a non-Abelian gauge theory pointing out some interesting features and physical implications.

In the next section we set up the basic framework for our discussion. The results are in Sec. III, divided into three subsections covering manifolds with disjoint boundaries, magnetic monopoles and realizations of the charge algebra. 


\section{BASIC FRAMEWORK}

As mentioned in the introduction, we will be focusing on the effects of nonzero boundary values for fields. To set the stage, we start with a brief discussion of the canonical setup for gauge theories. We are primarily interested in nonAbelian gauge theories, ignoring matter for the present. Thus the Lagrangian is that for the Yang-Mills theory and is given by

$$
\mathcal{L}=\frac{1}{4} \operatorname{Tr}\left(F_{\mu \nu} F^{\mu \nu}\right)=\frac{1}{2}\left(F_{0 i}^{a} F_{0 i}^{a}\right)-\frac{1}{4}\left(F_{i j}^{a} F_{i j}^{a}\right),
$$

where the fields strength tensor is, as usual, given by

$$
\begin{aligned}
F_{0 i} & =\left(-i T^{a}\right) F_{0 i}^{a}=\partial_{0} A_{i}-\partial_{i} A_{0}+\left[A_{0}, A_{i}\right], \\
F_{i j} & =\left(-i T^{a}\right) F_{i j}^{a}=\partial_{i} A_{j}-\partial_{j} A_{i}+\left[A_{i}, A_{j}\right] .
\end{aligned}
$$

Here $A_{0}$ and $A_{i}$ are Lie-algebra valued fields of the form $A_{0}=\left(-i T^{a}\right) A_{0}^{a}$ and $A_{i}=\left(-i T^{a}\right) A_{i}^{a}$, respectively; $\left\{T^{a}\right\}$ form a basis for the Lie algebra [which will be $S U(N)$ for most of our discussion]; they may be taken as hermitian matrices obeying the normalization $\operatorname{Tr}\left(T^{a} T^{b}\right)=\delta^{a b}$.

As is well known, the canonical momentum for $A_{i}$ is given by $E_{i}=F_{0 i}$, while the canonical momentum for $A_{0}$, which we may denote by $E_{0}$, is zero. This is to be viewed as a constraint imposed on the phase space made of all four fields and their momenta. The Hamiltonian is given by

$$
\begin{aligned}
\mathcal{H} & =\int d^{3} x\left(-\operatorname{Tr}\left(E_{i} \partial_{0} A_{i}+E_{0} \partial_{0} A_{0}\right)-\mathcal{L}\right) \\
& =\int d^{3} x\left[\frac{1}{2}\left(E_{i}^{a} E_{i}^{a}+B_{i}^{a} B_{i}^{a}\right)+E_{i}^{a}\left(D_{i} A_{0}\right)^{a}+E_{0}^{a} \Theta^{a}\right] .
\end{aligned}
$$

Since there is freedom of adding terms proportional to the constraints in the Hamiltonian, we can view $\partial_{0} A_{0}$ as an arbitrary Lie-algebra valued function. Put another way, there is nothing in the theory which determines $\partial_{0} A_{0}$; we denote this quantity by $\Theta$ in (3). If we eliminate the constraint $E_{0}$ and its corresponding conjugate constraint in the sense of Dirac's theory of constraints, this is equivalent to specifying $\partial_{0} A_{0}$. Another comment regarding the $E_{i} D_{i} A_{0}$ term is also in order. It arises from the canonical definition of the Hamiltonian and we have not done any integration by parts. This is important since we want to analyze boundary values and their effects. Secondly, the energy-momentum tensor for the Yang-Mills Lagrangian is given by

$$
T_{\mu \nu}=2 \frac{\delta S}{\delta g^{\mu \nu}}=\operatorname{Tr}\left(F_{\mu}^{\alpha} F_{\nu \alpha}\right)-g_{\mu \nu} \frac{1}{4} \operatorname{Tr}\left(F^{2}\right),
$$

where $S$ is the Yang-Mills action for an arbitrary metric $g_{\mu \nu}$ and after variation we set the $g_{\mu \nu}$ to be the Minkowski metric. If we now identify the Hamiltonian as the integral of $T_{00}$, then we do not have the $E_{i} D_{i} A_{0}$ term. Such differences between the canonical definition and what is defined by variation with respect to the metric can arise for fields which transform nontrivially under the Lorentz group. (We are referring to the Belinfante terms which are due to spin effects.) The Hamiltonian we are using in (3) is the standard canonical Hamiltonian.

We now define the operator

$$
Q(\mu)=\int d^{3} x E_{i}^{a}\left(D_{i} \mu\right)^{a}=-\int d^{3} x \operatorname{Tr}\left(E_{i} D_{i} \mu\right),
$$

where $\mu=\left(-i T^{a}\right) \mu^{a}$ is a test function, with $\mu^{a}$ being $\mathbb{R}$-valued functions. There are three types of test functions to be considered.

(1) If $\mu$ vanishes at spatial infinity, i.e., if $\mu^{a} \rightarrow 0$ as $|\vec{x}| \rightarrow \infty, Q(\mu)$ becomes the Gauss law operator $\mathcal{G}(\Lambda)$. The physical states are selected by the condition that they are annihilated by $\mathcal{G}(\Lambda$ ). (We use the letter $\Lambda$ for those functions $\mu$ which vanish at infinity.) Thus physical states obey

$$
\begin{aligned}
G(\Lambda) \mid \text { phys }\rangle & \left.=\int d^{3} x \operatorname{Tr}\left(E_{i} D_{i} \Lambda\right) \mid \text { phys }\right\rangle \\
& \left.=\int d^{3} x \operatorname{Tr}\left(-D_{i} E_{i}\right) \Lambda \mid \text { phys }\right\rangle=0 .
\end{aligned}
$$

(2) Now consider test functions $\mu \rightarrow 0$ as $|\vec{x}| \rightarrow \infty$, where the symbol $\rightarrow$ stands for "does not necessarily go to." The first situation is the case when $\mu$ does not vanish at infinity but is a constant, independent of angles, as $|\vec{x}| \rightarrow \infty$. We then define

$$
\begin{aligned}
\mathcal{G}_{0}= & \{\text { set of gauge transformations } g: \\
& \mathbb{R}^{3} \rightarrow S U(N), \text { such that } g(x) \rightarrow \text { const, } \\
& \text { not necessarily } 1 \text { as }|\vec{x}| \rightarrow \infty\}, \\
\mathcal{G}_{0}^{\infty}= & \{\text { set of gauge transformations } g: \\
& \mathbb{R}^{3} \rightarrow S U(N), \text { such that } g(x) \rightarrow 1 \\
& \text { as }|\vec{x}| \rightarrow \infty\} .
\end{aligned}
$$

Thus $\mathcal{G}(\Lambda)$ generate the group $\mathcal{G}_{0}^{\infty}$, while the $Q(\mu)$ 's generate the group $\mathcal{G}_{0}$. The quotient $\mathcal{G}_{0} / \mathcal{G}_{0}^{\infty}$ is the global group $G$, which we take to be $S U(N)$ for most of our discussions.

(3) More generally, we can have the situation where the test functions $\mu \rightarrow$ the angle-dependent limit; i.e., it is a nonconstant function on the two-sphere at spatial infinity. In this case, the operators $Q(\mu)$ generate the so-called Sky group [10].

The operators $Q(\mu)$ obey the commutation algebra

$$
\left[Q\left(\mu_{1}\right), Q\left(\mu_{2}\right)\right]=-i Q\left(\left[\mu_{1}, \mu_{2}\right]\right) .
$$


This is easily verified using the canonical equal-time commutation rules

$$
\left[A_{i}^{\alpha}(\vec{x}), E_{j}^{\beta}(\vec{y})\right]=i \delta^{\alpha \beta} \delta_{i j} \delta^{(3)}(\vec{x}-\vec{y}) .
$$

For the case of $\mu$ being a constant at spatial infinity, and on states obeying the Gauss law constraint (6), (7) reduces to $S U(N)$ Lie algebra.

We now briefly go back to the point made after (3) about $\partial_{0} A_{0}$. The constraint $E_{0} \approx 0$ is one of the primary constraints in the theory. Its time evolution can generate a secondary constraint. Consider the time evolution of $E_{0}$ smeared with a test function $\Theta$, with $\Theta \rightarrow 0$. The commutator with the Hamiltonian is given by

$$
\left[\int d^{3} x \operatorname{Tr}\left(E_{0} \Theta\right), \mathcal{H}\right]=i Q(\Theta) .
$$

This shows that the imposition of the constraint $\int \operatorname{Tr}\left(E_{0} \Theta\right)|\psi\rangle=0$ on the states would in turn require the vanishing of $Q(\Theta)$ where $\Theta \rightarrow 0$. In particular, this would mean that the charge carried by such states [measured by $Q(\Theta)$ for constant $\Theta$ ] could be zero. Thus charged states would be ruled out by fiat, if we impose $E_{0} \approx 0$ with a $\Theta$ which does not vanish at infinity. In eliminating the canonical pair $E_{0}, \Theta$, the boundary value of $\Theta=\partial_{0} A_{0}$ should not be eliminated if we allow charged states. This is consistent with the fact that the boundary values of $A_{0}$ and, hence, its time derivative can be chosen freely. They are externally chosen parameters which characterize the theory.

If $\Theta$ in (9) does go to zero at infinity, then we have the Gauss law operator on the right-hand side, which should annihilate physical states. Therefore there is no difficulty or inconsistency with requiring $\int \operatorname{Tr}\left(E_{0} \Theta\right) \approx 0$ for test functions which vanish at spatial infinity.

The conclusion is that, in general, the theory does allow for nonzero values for $A_{0}^{\infty}$ (and its time derivative). This is what we propose to analyze in subsequent sections.

So far we have argued that the mathematical framework allows for a nonzero $A_{0}$ at spatial infinity. We will now give two physical contexts where this does arise, before proceeding with further analysis.

First note that $A_{0} d t$ is the same in both Lorentzian and Euclidean cases as both $A_{0}$ and $d t$ acquire $i$ 's in passage to Euclidean signature. This means that some of the arguments about $A_{0} d t$ can be directly applied to finite temperature field theory where we have periodicity in Euclidean time, with period $\beta=1 / T$.

Now the gluons transform according to the adjoint representation $\operatorname{Ad} G$, which for $\mathrm{QCD}$ is $\operatorname{Ad}(S U(3))$. The fundamental group of $\operatorname{Ad} G$ is $\Pi_{1}(\operatorname{Ad}(S U(N)))=\mathbb{Z}_{N}$, so there are loops of gauge transformations $\tilde{g}(\vec{x}, t)$ which as $t$ varies from 0 to $\beta=1 / T$ wind from 1 to 1 nontrivially. [We may think of $t$ as (Euclidean) time, so that the loop is traced out as time evolves.] In $S U(N)$, the image of this set of transformations goes from $g(\vec{x}, 0)=\mathbf{1}$ to $g(\vec{x}, \beta)=z$, where $z$ is an element of $\mathbb{Z}_{N}$; i.e., $z \in\left(1, e^{2 \pi i / N}, e^{4 \pi i / N}\right.$, $\left.\ldots, e^{2(N-1) \pi i / N}\right) \times \mathbf{1}$, where $\mathbf{1}$ is the $N \times N$ identity matrix.

Such a curve in $S U(N)$ cannot, in general, become a constant in $t$ as $|\vec{x}| \rightarrow \infty$ in $g(\vec{x}, t)$ since the end points of the curve are fixed to be $\mathbf{1}$ and $z \neq \mathbf{1}$. Hence

$$
\lim _{|\vec{x}| \rightarrow \infty} \partial_{0} g(\vec{x}, t) \neq 0 \quad \text { identically. }
$$

Thus, even if we start with $A_{0}^{\infty}=0$, a gauge transformation by such an element can lead to a nonzero $A_{0}^{\infty}=$ $\left.g^{-1} \partial_{0} g\right]_{|\vec{x}| \rightarrow \infty} \neq 0$. It is well known that such gauge transformations are important in finite temperature field theory with the Polyakov loop

$$
L=\operatorname{Tr} \exp \left(i \int_{0}^{\beta} d t A_{0}(\vec{x}, t)\right)
$$

considered as an order parameter. There are also indications from numerical work that the expectation value of $L$ need not be the identity at high temperatures, consistent with the possibility that $A_{0}^{\infty}$ can be nonzero.

Our second example is a classic one, the Josephson effect. The basic setup here is that one has a normal conductor sandwiched between two superconductors. The normal material constitutes a spacetime region with disjoint boundaries. Thus the electrons in the normal material can be described by electrodynamics for a region with boundaries. Generally we can have different phases for the many-body wave function in the two superconducting regions. The phases of the electron at the boundaries of the normal material have to be matched to these. The difference of phases leads to the Josephson current, with the time derivative of the phase acting as the value of $A_{0}$ on the boundary. This is another case of a gauge theory in a physical context with nonzero values of $A_{0}$ on the boundaries.

\section{PHYSICAL IMPLICATIONS OF BOUNDARY DATA}

We now consider some physical implications of nontrivial boundary data. We will consider two examples: a field theory defined on a manifold with disjoint boundaries, and chemical potentials for non-Abelian charges in the presence of magnetic monopoles.

\section{A. Manifolds with disjoint boundaries}

The simplest case would be to start with a non-Abelian gauge theory, say, QCD, in $1+1$ dimensions, with the real line as the spatial manifold. In this case we have disjoint boundaries corresponding to $|\vec{x}| \rightarrow \pm \infty$. Let $A_{0}^{ \pm \infty}$ denote the two boundary values of $A_{0}$; i.e., $A_{0} \rightarrow A_{0}^{ \pm \infty}$ as $|\vec{x}| \rightarrow \pm \infty$, respectively. We can then decompose $A_{0}$ as 


$$
\begin{aligned}
& A_{0}=A_{0}^{+}+A_{0}^{-}, \\
& A_{0}^{ \pm} \rightarrow A_{0}^{ \pm \infty} \quad \text { as }|\vec{x}| \rightarrow \pm \infty, \\
& A_{0}^{ \pm} \rightarrow 0 \quad \text { as }|\vec{x}| \rightarrow \mp \infty .
\end{aligned}
$$

This is essentially a decomposition of $A_{0}$ via a resolution of unity over the real line. The specific choice of $A_{0}^{ \pm}$is not important; different choices with the same boundary values differ by terms proportional to the Gauss law constraint. In what follows, we will, for simplicity, consider the case with $\partial_{0} A_{0}^{ \pm} \rightarrow 0$ as $|\vec{x}| \rightarrow \pm \infty$; i.e., the boundary values of $A_{0}$ are taken to be independent of time. For physical states $\mid$ phys $\rangle$ which obey the Gauss law, we have

$$
\begin{aligned}
Q\left(A_{0}\right) & =Q\left(A_{0}^{+}\right)+Q\left(A_{0}^{-}\right), \\
{\left.\left[Q\left(A_{0}^{+}\right), Q\left(A_{0}^{-}\right)\right] \mid \text {phys }\right\rangle } & =0 .
\end{aligned}
$$

Effectively we have two operators $Q\left(A_{0}^{ \pm}\right)$associated to charges defined via fluxes on the two disjoint boundaries. Since the Hamiltonian has a term $Q\left(A_{0}\right)$, once a choice of $A_{0}^{ \pm \infty}$ has been made, the residual symmetry of the theory is given by the common stability group of $Q\left(A_{0}^{ \pm}\right)$. In general, the stability groups for these two operators $Q\left(A_{0}^{ \pm}\right)$can be different. Transformations corresponding to the charge algebra, with parameters $\theta$, are generated by $Q(\theta)=$ $\int \operatorname{Tr}(E D \theta)$. Analogous to how $A_{0}$ was decomposed, we can write

$$
\begin{aligned}
\theta & =\theta^{+}+\theta^{-}, \\
\theta^{ \pm} & \rightarrow \theta^{ \pm \infty} \quad \text { as }|\vec{x}| \rightarrow \pm \infty, \\
\theta^{ \pm} & \rightarrow 0 \quad \text { as }|\vec{x}| \rightarrow \mp \infty .
\end{aligned}
$$

This leads to two copies of the algebra of charges, corresponding to $Q\left(\theta^{+}\right)$and $Q\left(\theta^{-}\right)$.

As mentioned above, the boundary values and hence the stability groups of $Q\left(A_{0}^{ \pm} \infty\right)$ can be different for different disjoint components of the boundary. Two examples of this for $S U(3)$ would be the choices

$A_{0}^{+\infty}=a_{0}^{+\infty} \operatorname{diag}(1,1,-2), \quad A_{0}^{-\infty}=a_{0}^{-\infty} \operatorname{diag}(1,-2,1)$,

$A_{0}^{+\infty}=a_{0}^{+\infty} \operatorname{diag}(1,1,-2), \quad A_{0}^{-\infty}=b_{0}^{-\infty} \operatorname{diag}(1,-1,0)$.

One of the physical consequences of such unequal choices on disjoint boundaries is that it can lead to a current. This is similar to how a tunneling current arises in the Josephson junction mentioned earlier. To demonstrate how this can happen, we consider the theory with the addition of fields which carry nonzero charges. For simplicity, we take them to be Dirac fields, so that the action written out in first order form is

$$
\begin{aligned}
S= & \int\left[E_{i}^{a} \dot{A}_{i}^{a}-\frac{1}{2}\left(E_{i}^{a} E_{i}^{a}+B_{i}^{a} B_{i}^{a}\right)-E_{i}^{a}\left(D_{i} A_{0}\right)^{a}+i \bar{\psi} \gamma_{0} \partial_{0} \psi\right. \\
& \left.-i \bar{\psi} \gamma_{i} \partial_{i} \psi+i \bar{\psi} \gamma_{0} A_{0} \psi-i \bar{\psi} \gamma_{i} A_{i} \psi\right] .
\end{aligned}
$$

We can now write $A_{0}=g^{-1} \dot{g}$ for some group element $g$ which is constant on each connected piece of the boundary at fixed $t$. Using this we can rewrite the action (16) as

$$
\begin{aligned}
S= & \int\left[\mathcal{E}_{i}^{a} \dot{\mathcal{A}}_{i}^{a}-\frac{1}{2}\left(\mathcal{E}_{i}^{a} \mathcal{E}_{i}^{a}+\mathcal{B}_{i}^{a} \mathcal{B}_{i}^{a}\right)+i \bar{\psi} \gamma_{0} \partial_{0} \psi-i \bar{\psi} \gamma_{i} \partial_{i} \psi\right. \\
& \left.+i \bar{\psi} \gamma_{0} g^{-1} \dot{g} \psi-i \bar{\psi} \gamma_{i}\left(g^{-1} \mathcal{A}_{i} g+g^{-1} \partial_{i} g\right) \psi\right]
\end{aligned}
$$

where

$$
\mathcal{A}_{i}=g A_{i} g^{-1}-\partial_{i} g g^{-1}
$$

with $\mathcal{E}_{i}^{a}$ as its canonical conjugate, and $\mathcal{B}$ and $\mathcal{D}$ are constructed using $\mathcal{A}$. We can take $\mathcal{A}_{i}$ and $\mathcal{E}_{i}$ as the basic phase space variables for the gauge field. For this choice of variables, the $g$ dependence is transferred entirely to the matter terms. The dependence of the partition function or the functional integral on the boundary values can be obtained by varying $g$. With $g^{-1} \delta g=-i t^{a} \delta \theta^{a}$, we find

$$
\begin{aligned}
\delta S & =\delta S_{\text {surf }}+\int\left[-\left(D_{0} J_{0}\right)^{a}+\left(D_{i} J_{i}\right)^{a}\right] \delta \theta^{a}, \\
\delta S_{\text {surf }} & \left.=\int_{V} \bar{\psi} \gamma_{0} t^{a} \psi \delta \theta^{a}\right]_{t_{i}}^{t_{f}}-\int_{\partial V} \bar{\psi} \gamma_{i} t^{a} \psi \delta \theta^{a} d S_{i} d t, \\
J_{0}^{a} & =\bar{\psi} \gamma_{0} t^{a} \psi, \quad J_{i}^{a}=\bar{\psi} \gamma_{i} t^{a} \psi .
\end{aligned}
$$

We are considering the fields in a spatial volume $V$ with the time interval as $\left[t_{f}, t_{i}\right]$. The boundary $\partial V$ can have disjoint components. The last set of terms in (19) will vanish in the classical case by current conservation. In the quantum theory, the conservation law, which follows from variation of the action, can be imposed as an operator equation inside matrix elements or at the level of the integrand of the functional integral. To see this in more detail, consider the vacuum-to-vacuum transition amplitude for a set of fields generically denoted as $\varphi$ :

$$
Z \equiv\langle 0 \mid 0\rangle=\int[d \varphi] \Psi_{0}^{*}\left[\varphi_{f}, t_{f}\right] e^{i S\left(\varphi, t_{f}, t_{i}\right)} \Psi_{0}\left[\varphi_{i}, t_{i}\right],
$$

where $S$ is the action evaluated for field configurations $\varphi(\vec{x}, t)$ with $\varphi\left(\vec{x}, t_{f}\right)=\varphi_{f}, \varphi\left(\vec{x}, t_{i}\right)=\varphi_{i}$. We integrate over $\varphi_{f}$ and $\varphi_{i}$ as well with the wave functions as the weighting factors. Consider the functional derivative with respect to the bulk values of the fields, which keep $\varphi_{f}$ and $\varphi_{i}$ and the spatial boundary value of $\varphi(\vec{x}, t)$ fixed. The vanishing of the integral of a total derivative then leads to the identity 


$$
\begin{aligned}
0 & =\int[d \varphi] \Psi_{0}^{*}\left[\varphi_{f}, t_{f}\right]\left[\frac{\delta}{\delta \varphi} e^{i S}\right] \Psi_{0}\left[\varphi_{i}, t_{i}\right] \\
& =i \int[d \varphi] \Psi_{0}^{*}\left[\varphi_{f}, t_{f}\right] e^{i S} \frac{\delta S}{\delta \varphi} \Psi_{0}\left[\varphi_{i}, t_{i}\right]
\end{aligned}
$$

(If we have sources, there will be additional terms corresponding to their transformation; this is standard procedure for the derivation of Ward-Takahashi identities.) This shows how the bulk equations of motion can be realized in matrix elements. For the more general variation of the vacuum-to-vacuum amplitude with no sources, we can then write

$$
\begin{aligned}
Z^{\prime} & =Z+\int \delta \theta^{a} \frac{\delta Z}{\delta \theta^{a}} \\
& =\int[d \varphi] \Psi_{0}^{*}\left[\varphi_{f}+\delta \varphi_{f}, t_{f}\right] e^{i(S+\delta S)} \Psi_{0}\left[\varphi_{i}+\delta \varphi_{i}, t_{i}\right] \\
& =\int[d \varphi] \Psi_{0}^{*}\left[\varphi_{f}+\delta \varphi_{f}, t_{f}\right] e^{i S} e^{i \delta S_{\text {sur }} \Psi_{0}\left[\varphi_{i}+\delta \varphi_{i}, t_{i}\right]} .
\end{aligned}
$$

The surface terms in $\delta S_{\text {surf }}$ on the two time slices at $t_{f}$ and $t_{i}$ can be absorbed into the change of the final and initial wave functions, since the wave functions transform as $\delta \Psi(\varphi, t)=i\left[\delta S_{\text {surf }}\right]_{\text {at } t} \Psi(\varphi, t)$. Thus, upon using (19) and (21), we can simplify (22) as

$$
\int \delta \theta^{a} \frac{\delta Z}{\delta \theta^{a}}=-i \int[d \varphi] \Psi_{0}^{*}\left[\varphi_{f}, t_{f}\right] e^{i S}\left[\sum F_{\alpha}^{a} \delta \theta_{\alpha}^{a}\right] \Psi_{0}\left(\varphi_{i}, t_{i}\right),
$$

where $F_{\alpha}^{a}=\int_{\partial V_{\alpha}} J_{i}^{a} d S_{i}$ is the flux of the current across each connected component (indexed by $\alpha$ ) of the spatial boundary. These arguments go through in Euclidean signature with $Z$ being the partition function.

Now consider calculating the partition function (or the vacuum-to-vacuum amplitude) $Z$ using the action as given in (16). The result will obviously depend on the boundary values of $A_{0}$, or, equivalently, on $g$. So the variation of $Z$ with respect to $g$ is, in general, not zero. The second way of calculating involves using the action (17). In this case, the dependence of $Z$ on $g$ is given by the expectation value of the current fluxes as in (23). The compatibility of these two ways of calculating $Z$ tells us that if the functional integral or partition function depends on the boundary values of $A_{0}$, then there must be current fluxes across the boundaries. Notice that, with disjoint boundaries, we can vary $g$ independently on the different connected components, and so the individual fluxes cannot be zero.

Although we started with the $1+1$-dimensional case, this part of the discussion [starting with (16)] is general and applies to theories in higher dimensional manifolds with disjoint boundaries. To summarize, if we have different values of $A_{0}$ on different disconnected pieces of the boundary, there will be current fluxes in the bulk of spatial volume. We also note that if the operators which commute with the boundary data form a non-Abelian algebra, in the quantum theory, they are associated with a multiplicity of edge states. As these commutants can differ at the different edges, these multiplicities also can differ.

We have already remarked on the similarity of what we do here and the tunneling current in a Josephson junction. A physical situation where our arguments for the nonAbelian case would apply is for neutron stars. The general expectation is that in the interior of a neutron star there are concentric layers of hadronic matter in different phases [11]. These include pure quark phase, quark slabs, etc. The interfaces of such regions function as the disjoint boundaries of our discussion above. It is difficult to make more specific predictions at this stage, since the physics of the hadronic phases in the neutron star is still not well understood.

\section{B. Magnetic monopoles}

In the previous example, we considered $A_{0}$ to be a constant on the boundary. But there can be topological obstructions to this choice. The simplest example which can illustrate this is the case of a background corresponding to the 't Hooft-Polyakov monopole. Recall that the Higgs field $\Phi$ in this case has the asymptotic behavior

$$
\Phi_{|\vec{x}| \rightarrow \infty} \rightarrow \Phi^{\infty}(\hat{x})=f_{0}^{\infty} \vec{\tau} \cdot \hat{\phi}(\hat{x}),
$$

where the winding number of the map $\hat{x} \rightarrow \hat{\phi}(\hat{x})$ is the monopole charge and $f_{0}^{\infty}$ is a constant. $\left[\tau^{a}\right.$ are the Pauli matrices, viewed as the generators of the Lie algebra of $S U(2)$.]

The Hamiltonian (3) has the term $Q\left(A_{0}\right)$ in addition to the usual bulk terms. The boundary value of $A_{0}$ must be such that the Hamiltonian commutes with the Higgs field, so that the value of the Higgs field at spatial infinity is not changed by time evolution. This is needed for the chosen field configuration to correspond to a sector with spontaneously broken symmetry (and a static monopole field). Specifically, we must require that

$$
\left[Q\left(A_{0}\right), \Phi\right]_{|\vec{x}| \rightarrow \infty} \rightarrow 0 .
$$

If $A_{0}^{\infty}(\hat{x})$ is the boundary value, i.e., $A_{0}(\vec{x})_{|\vec{x}| \rightarrow \infty} \rightarrow A_{0}^{\infty}(\hat{x})$, the requirement (25) leads to

$$
A_{0}^{\infty}(\hat{x})=a_{0}^{\infty} \vec{\tau} \cdot \hat{\phi}(\hat{x}),
$$

where $a_{0}^{\infty}$ is a constant. Thus we cannot choose $A_{0}$ to be a constant on the two-sphere at spatial infinity; it depends on $\vec{x}$ as indicated. $A_{0}^{\infty}(\hat{x})$ may be viewed as the chemical potential for non-Abelian charges in the presence of the 
monopole. The term in the Hamiltonian to be used to define the partition function for a system with finite charge is thus

$$
Q\left(A_{0}\right)=\int d^{3} x \operatorname{Tr}\left(E_{i} D_{i} A_{0}\right)
$$

with $A_{0}^{\infty}(\hat{x})$ as in (26). On quantum states, because of the Gauss law, $Q\left(A_{0}\right)$ depends only on the equivalence class $\left[A_{0}\right]$. Thus if $A_{0}^{(i)} \in\left[A_{0}\right]$, then $\left(A_{0}^{(1)}-A_{0}^{(2)}\right)_{|\vec{x}| \rightarrow \infty} \rightarrow 0$.

An additional feature or nuance associated to this can be seen by considering the case of the grand unified $S U(5)$ theory [12], broken down, as usual, to the standard model gauge group. In this case, the Higgs field $\Phi$ belongs to the representation 24 of $S U(5)$; this may be viewed as a traceless $5 \times 5$ matrix. In the nonmonopole sector, it breaks $S U(5)$ to $H=[S U(3) \times S U(2) \times U(1)] / \mathbb{Z}_{6}$; its asymptotic value on the two-sphere at spatial infinity is of the form

$$
\Phi^{\infty}=\phi^{\infty} \operatorname{diag}\left(1,1,1,-\frac{3}{2},-\frac{3}{2}\right),
$$

where $\phi^{\infty}$ is a constant. The $5^{*}$ matter multiplet is $\left(d_{1}^{c}, d_{2}^{c}, d_{3}^{c}, e^{-}, \nu_{e}\right)_{L}$. Thus, we see clearly that (28) leaves color rotations of the three $d^{c}$ 's invariant, and similarly for the $S U(2)$ rotations on $\left(e^{-}, \nu_{e}\right)$. Defining $T_{3}=\frac{1}{2} \operatorname{diag}$ $(0,0,1,-1,0)$, we can write the asymptotic value of the field as

$$
\Phi^{\infty}=\phi^{\infty}\left[\frac{5}{2} T_{3}+\operatorname{diag}\left(1,1,-\frac{1}{4},-\frac{1}{4},-\frac{3}{2}\right)\right] .
$$

The reason for writing it this way is that we can regard the $S U(5)$ monopole as the 't Hooft-Polyakov monopole of an $S U$ (2) subgroup acting on, say, $\left(d_{3}^{c}, e^{-}\right)$broken down to a $U(1)$. Thus, for a generic monopole ansatz, the asymptotic value of the Higgs field can be obtained by rotating (29) over the sphere $S_{\infty}^{2}$ at infinity. In the lowest monopole sector, it is given by

$\Phi^{\infty}(\hat{x})=\phi^{\infty}\left[\frac{5}{2} T \cdot \hat{x}+\operatorname{diag}\left(1,1,-\frac{1}{4},-\frac{1}{4},-\frac{3}{2}\right)\right]$.

For a general winding number solution, we can replace $\hat{x}$ by $\hat{\phi}(\hat{x})$ in (30) where $\hat{\phi}(\hat{x})$ is as in (24).

The $S U(5)$ monopoles are non-Abelian. From the structure of the spontaneous symmetry breaking, it would seem that one can introduce color $S U(3)$ rotations and $S U(2) \times U(1)$ rotations as collective coordinates or moduli whose quantization would convert the classical solution into a suitable multiplet of $H=[S U(3) \times S U(2) \times$ $U(1)] / \mathbb{Z}_{6}$. However, it is known that this cannot be done [13]. There is a topological obstruction, and typically, in the monopole sector, the unbroken subgroup $H^{\prime}$ is smaller than $H$ and depends on the choice of the monopole solution. For the asymptotic behavior given in (30), the unbroken group is $H^{\prime}=U(2) \times U(1) \times U(1)$. The full theory is discussed in $[14,15]$.

Now consider a gas of gluons, $W$ 's and other particles carrying non-Abelian charges in the presence of a monopole characterized by, say, (30). For a statistical distribution of these particles with nonzero charge, we need the chemical potential. However, as mentioned above, it must commute with the Higgs field of the monopole. So $A_{0}^{\infty}$, to be used as the chemical potential, can be any element in the Lie algebra of $U(2) \times U(1) \times U(1)$.

The older work [13-15] shows the topological obstruction to color and $S U(2)$ rotations of the monopole. The conclusion of the present argument is that this breakdown of color in the presence of such monopoles applies also to forming statistical distributions of the charged particles on this background.

Regarding the statistical distributions of particles, it may also be interesting to note that some of the particles can undergo a change of statistics. Notice that the Higgs field in (30) is invariant under a simultaneous rotation of $\hat{x}_{i}$ and $T_{i}$. This means that the angular momentum operator in this background will be a combination $L_{i}+S_{i}+T_{i}$, where $L_{i}$ and $S_{i}$ denote the orbital and spin angular momentum operators, respectively. This is the well-known "spin from isospin" in a monopole background [16]. For the present context this means that the particles corresponding to $\left(d_{3}^{c}, e^{-}\right)$will change statistics. Many of the gauge bosons become massive at the scale of $S U(5)$ breaking. The gauge particles which remain massless at this stage will retain their statistics since they transform as the adjoint representation of the charge algebra and hence the contributions from $T_{i}$ will be integer valued.

\section{The realization of the charge algebra}

We will now make some comments on how the charge operators act on the states, i.e., on the nature of the representation of the charge algebra. These statements are based on the Fabri-Picasso [17] theorem and are basically the application of that result to the present context.

We note however that these theorems require the existence of a mass gap separating vacuum and the excited states [18]. There is no rigorous proof that there is such a mass gap in non-Abelian gauge theories.

Consider the charge operators given in (5). The Hamiltonian has a term $Q\left(A_{0}\right)$ and we have the commutation rule $[Q(\mu), \mathcal{H}]=-i Q\left(\left[\mu, A_{0}\right]\right)$. By the Gauss law, only the boundary value of the commutator $\left[\mu, A_{0}\right]$ is relevant when this relation acts on physical states. Thus the commutant of $A_{0}^{\infty}$ in the Lie algebra defines the symmetry group of the problem, which we will refer to as $G_{A_{0}}$ for brevity.

The momentum operator $P_{i}$ is a crucial ingredient for the Fabri-Picasso theorem, so it is useful to consider the expression for it in our case. Explicitly, 


$$
P_{i}=\int d^{3} x E_{j}^{a} F_{i j}^{a}
$$

If $\xi_{i}$ is a constant vector, we can write

$$
\xi_{i} P_{i}=\int d^{3} x E_{i}^{a}(\xi \cdot \partial) A_{i}^{a}-\int d^{3} x E_{i}^{a} D_{i}(A \cdot \xi) .
$$

The last term will generate a gauge transformation with parameter $A \cdot \xi$, if we take $A_{i}$ to vanish at the spatial boundary. It is then clear from the first term that $P_{i}$ generates translations on all gauge-invariant quantities or on operators acting on physical states. From the commutation rule (8), we can easily check that

$$
\left[Q(\mu), P_{i}\right]=0 .
$$

It should be emphasized that this relation holds even for functions $\mu$ which do not vanish on the spatial boundary or spatial infinity. No integration by parts or discarding of boundary terms is needed.

We also note that the charge operator $Q(\mu)$, with a constant value for $\mu$ at the boundary, can be written in terms of a density using the Gauss law. On physical states, we can write

$$
Q(\mu)|\mathrm{phys}\rangle=\oint \mu^{a} E_{i}^{a} d S_{i}|\mathrm{phys}\rangle .
$$

Integrating $\mu^{a}\left(\nabla \cdot E^{a}+f^{a b c} A_{i}^{b} E_{i}^{c}\right) \mid$ phys $\rangle=0 \quad$ over all space and taking $\mu^{a}$ to be a constant equal to the value on the boundary, we find

$$
\begin{aligned}
Q(\mu) \mid \text { phys }\rangle & \left.=-\int d^{3} x\left(\mu^{a} f^{a b c} A_{i}^{b} E_{i}^{c}\right) \mid \text { phys }\right\rangle \\
& \left.\equiv \int d^{3} x J_{0}(x) \mid \text { phys }\right\rangle .
\end{aligned}
$$

We can now see the Fabri-Picasso theorem for this case as follows:

$$
\left\langle 0\left|J_{0}(x) Q(\mu)\right| 0\right\rangle=\left\langle 0\left|e^{i P \cdot x} J_{0}(0) e^{-i P \cdot x} Q(\mu)\right| 0\right\rangle=0
$$

using (33) and $P_{i}|0\rangle=0$. This immediately gives

$$
\begin{aligned}
\langle 0|Q(\mu) Q(\mu)| 0\rangle & =\int d^{3} x\left\langle 0\left|J_{0}(0) Q(\mu)\right| 0\right\rangle \\
& =\text { const } \times \int d^{3} x \\
& =\text { const } \times \infty .
\end{aligned}
$$

This result shows that either $Q(\mu)|0\rangle=0$ (corresponding to the vanishing of the constant in this equation) or the state $Q(\mu)|0\rangle$ does not exist as it is not normalizable.

So far, this discussion is similar to what happens with spontaneous symmetry breaking. But for us, there is an additional condition because the Hamiltonian contains a term which is $Q\left(A_{0}\right)$. Thus $H|0\rangle$ produces a term $Q\left(A_{0}\right)|0\rangle$ and if this is not normalizable, the vacuum state will not be in the domain of the Hamiltonian. So the only physically sensible choice is $Q\left(A_{0}\right)|0\rangle=0$. Notice that this also tells us that the vacuum energy is not shifted by the presence of the term $Q\left(A_{0}\right)$. Now consider those $Q(\mu)$ which weakly commute with $Q\left(A_{0}\right)$, with $\mu \neq A_{0}$. These elements, along with $Q\left(A_{0}\right)$ generate the subgroup $G_{A_{0}}$. For these $Q(\mu)$, there is no real restriction; either choice $[Q(\mu)|0\rangle=0$ or $Q(\mu)|0\rangle \neq 0$ ] is possible. Thus we can have a situation where $G_{A_{0}}$ is fully realized as a symmetry or partially or fully broken spontaneously to the $U(1)$ group generated by $Q\left(A_{0}\right)$.

Now let us turn to the choices of $\mu$ with $[Q(\mu)$, $\left.Q\left(A_{0}\right)\right]=-i Q\left(\left[\mu, A_{0}\right]\right) \neq 0$. These would generate elements of the gauge group $G$ which are not contained in $G_{A_{0}}$ and hence are not symmetries of the theory. Again, there are two possibilities: either $Q(\mu)|0\rangle=0$ (with $\left[\mu, A_{0}^{\infty}\right] \neq 0$ ) or its action on $|0\rangle$ is not defined following the argument given above. Coleman's theorem [19] would suggest that the case $Q(\mu)|0\rangle=0$ can lead to additional symmetries and hence should be ruled out since all $Q(\mu)$ which commute with the Hamiltonian have been included as generators of $G_{A_{0}}$. But it is not clear that the premises of Coleman's theorem (which include Lorentz invariance and the Reeh-Schlieder theorem) are obtained in our case.

To summarize, there is always an unbroken $U(1)$ subgroup generated by $Q\left(A_{0}\right)$; one may have a larger symmetry $G_{A_{0}}$, which may be partially or fully spontaneously broken down to this $U(1)$. (Since we are focusing only on the Yang-Mills action, the explicit mechanism for such a breaking is not manifest; we would have to include additional charged fields to analyze this.) Transformations corresponding to elements of the global group $G$ which are not in $G_{A_{0}}$ are not symmetries of the theory.

Another point of interest is that the term $Q\left(A_{0}\right)$ in the Hamiltonian can lead to energy corrections to the charged states. Writing $\mathcal{H}=\mathcal{H}_{0}+Q\left(A_{0}\right)$, notice that $\mathcal{H}_{0}$ and $Q\left(A_{0}\right)$ commute with each other and can be simultaneously diagonalized. A nonzero $A_{0}^{\infty}$ can lead to splitting of degenerate states for the charged particles like gluons, charge being defined by $Q\left(A_{0}\right)$. We also note that while there are many issues associated with the implementation of non-Abelian charge rotations on the charged sector in general [9], in the present case, we are only dealing with an Abelian group defined by $Q\left(A_{0}\right)$.

\section{ACKNOWLEDGMENTS}

This research was supported in part by the U.S. National Science Foundation Grant No. PHY-1820721 and by Professional Staff Congress-City University of New York (PSC-CUNY) awards. 
[1] D. Birmingham, M. Blau, M. Rakowski, and G. Thompson, Phys. Rep. 209, 129 (1991); A. S. Schwarz, arXiv:hep-th/ 0011260.

[2] See e.g., M. Stone, Quantum Hall Effect (World Scientific, Singapore, 1992), Chap. 5; D. Karabali and V. P. Nair, J. Phys. A 39, 12735 (2006); for an early treatment of edge states for the Chern-Simons theory, see E. Witten, Commun. Math. Phys. 121, 351 (1989); for conformal edge currents in Chern-Simons theories, see A.P. Balachandran, G. Bimonte, K. S. Gupta, and A. Stern, Int. J. Mod. Phys. A 07, 4655 (1992); see also the paper by Balachandran et al. in [7]; the Chern-Simons source as a conformal family and its vertex operators are discussed in A. P. Balachandran, G. Bimonte, K. S. Gupta, and A. Stern, Int. J. Mod. Phys. A 07, 5855 (1992).

[3] For general reviews on the Casimir effect, see K. A. Milton, J. Phys. Conf. Ser. 161, 012001 (2009); The Casimir Effect: Physical Manifestations of Zero-Point Energy (World Scientific, Singapore, 2001); M. Bordag, U. Mohideen, and V. M. Mostepanenko, Phys. Rep. 353, 1 (2001); M. Bordag, G. L. Klimchitskaya, U. Mohideen, and V. M. Mostepanenko, Advances in the Casimir Effect, International Series of Monographs on Physics (Oxford University Press, Oxford, 2009).

[4] A. P. Balachandran, G. Bimonte, K. S. Gupta, and A. Stern, Int. J. Mod. Phys. A 07, 4655 (1992); 10, 1969 (1995); N. Acharyya, A. P. Balachandran, V. E. Díez, P. N. B. Subramanian, and S. Vaidya, Phys. Rev. D 94, 085026 (2016).

[5] M. Asorey, A. Ibort, and G. Marmo, Int. J. Mod. Phys. A 20, 1001 (2005); M. Asorey and J. M. Munoz-Castañeda, J. Phys. A 41, 304004 (2008); M. Asorey, D. Garcia-Alvarez, and J. M. Munoz-Castañeda, J. Phys. A 39, 6127 (2006); 40, 6767 (2007); A. P. Balachandran, L. Chandar, E. Ercolessi, T. R. Govindarajan, and R. Shankar, Int. J. Mod. Phys. A 09, 3417 (1994); M. Asorey and J. M. Munoz-Castañeda, Nucl. Phys. B874, 852 (2013); M. Asorey, A. P. Balachandran, and J. M. Perez-Pardo, Rev. Math. Phys. 28, 1650020 (2016).

[6] T. R. Govindarajan and R. Tibrewala, Phys. Rev. D 83, 124045 (2011); T. R. Govindarajan and V. P. Nair, Phys. Rev. D 89, 025020 (2014); T. R. Govindarajan and R. Tibrewala, Phys. Rev. D 92, 045040 (2015).
[7] D. N. Kabat, Nucl. Phys. B453, 281 (1995); A. P. Balachandran, L. Chandar, and A. Momen, Int. J. Mod. Phys. A 12, 625 (1997), and references therein; W. Donnelly and A. C. Wall, Phys. Rev. Lett. 114, 111603 (2015); Phys. Rev. D 94, 104053 (2016); H. Casini and M. Huerta, Phys. Rev. D 93, 105031 (2016); C. A. Agon, M. Headrick, D. L. Jafferis, and S. Kasko, Phys. Rev. D 89, 025018 (2014); H. J. Schnitzer, arXiv:1611.03116; A. Agarwal, D. Karabali, and V. P. Nair, Phys. Rev. D 96, 125008 (2017).

[8] J. Fröhlich, G. Morchio, and F. Strocchi, Ann. Phys. (N.Y.) 119, 241 (1979); Phys. Lett. 89B, 61 (1979); J. Fröhlich, Commun. Math. Phys. 66, 223 (1979); D. Buchholz, Commun. Math. Phys. 85, 49 (1982); Phys. Lett. B 174, 331 (1986).

[9] A. P. Balachandran, A. de Queiroz, and S. Vaidya, Int. J. Mod. Phys. A 30, 1550064 (2015); A. P. Balachandran, S. Vaidya, and A. R. de Queiroz, Mod. Phys. Lett. A 30, 1550080 (2015); A. P. Balachandran, Mod. Phys. Lett. A 31, 1650060 (2016); A. P. Balachandran and V. P. Nair, Phys. Rev. D 98, 065007 (2018).

[10] A. P. Balachandran and S. Vaidya, Eur. Phys. J. Plus 128, 118 (2013).

[11] N. K. Glendenning, Compact Stars: Nuclear Physics, Particle Physics and General Relativity, 2nd ed. (Springer, New York, 2000).

[12] See, e.g., G. Ross, Grand Unified Theories (AddisonWesley Publishing, New York, 1984).

[13] A. P. Balachandran, G. Marmo, N. Mukunda, J. S. Nilsson, E. C. G. Sudarshan, and F. Zaccaria, Phys. Rev. Lett. 50, 1553 (1983); P. C. Nelson and A. Manohar, Phys. Rev. Lett. 50, 943 (1983).

[14] A. P. Balachandran, G. Marmo, N. Mukunda, J. S. Nilsson, E. C. G. Sudarshan, and F. Zaccaria, Phys. Rev. D 29, 2919 (1984); 29, 2936 (1984).

[15] P. C. Nelson and S. R. Coleman, Nucl. Phys. B237, 1 (1984).

[16] R. Jackiw and C. Rebbi, Phys. Rev. Lett. 36, 1116 (1976).

[17] E. Fabri and L. E. Picasso, Phys. Rev. Lett. 16, 408 (1966).

[18] See, e.g., C. A. Orzalesi, Rev. Mod. Phys. 42, 381 (1970).

[19] S. Coleman, J. Math. Phys. (N.Y.) 7, 787 (1966). 